\title{
Oxidation processes of Longissimus dorsi from pigs supplemented with linseed oil and antioxidants
}

\author{
M. Brodowska ${ }^{1 \#}$, D. Guzek ${ }^{1}$, J. Godziszewska ${ }^{1}$, E. Pogorzelska $^{1}$ \& A. Wierzbicka ${ }^{2}$ \\ ${ }^{1}$ Laboratory of Food Chemistry, Faculty of Human Nutrition and Consumer Sciences, Warsaw University of Life Sciences \\ (WULS - SGGW), Nowoursynowska str159 c, 02-776 Warsaw, Poland \\ ${ }^{2}$ Department of Technique and Food Development, Faculty of Human Nutrition and Consumer Sciences, Warsaw \\ University of Life Sciences (WULS - SGGW), Nowoursynowska str 159 c, 02-776 Warsaw, Poland
}

(Received 3 August 2017; Accepted 26 January 2018; First published online 21 June 2018)

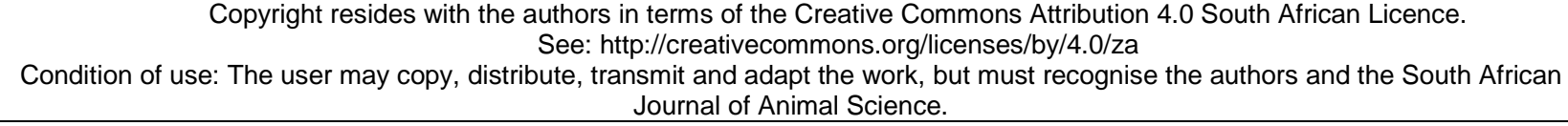

\begin{abstract}
The aim of this study was to determine the effects of pig fodder supplementation on lipid oxidation of Longissimus dorsi ( L. dorsi ) after frozen storage at $-20^{\circ} \mathrm{C} \pm 1{ }^{\circ} \mathrm{C}$ for nine months. Fodder additives included $3 \%$ linseed oil (L1) or 3\% linseed oil and antioxidants containing $100 \mathrm{mg}$ vitamin E/kg and $1 \mathrm{mg}$ organic selenium $/ \mathrm{kg}(\mathrm{L} 2)$. The oxidation processes were evaluated by measuring thiobarbituric acid reactive substances (TBARS) and analyses of a profile of volatile organic compounds (VOC). The VOC were determined using an electronic nose based on ultra-fast gas chromatography. The level of TBARS for diets was $1.88 \pm 0.52$ to $2.30 \pm 1.10 \mathrm{mg}$ malondialdehyde/ $\mathrm{kg}$ of meat. The results indicated that the diet of pigs from L1 and L2 groups had no impact on the TBARS value of $L$. dorsi pork frozen for nine months. On the other hand, aldehydes, which are regarded as compounds characteristic of oxidation processes, were identified in all samples. Volatile aldehydes contributed approximately $10 \%, 12 \%$, and $15 \%$ of total detected volatiles for L2, L1, and the control group, respectively. Moreover, the data showed that propanal and benzeneacetaldehyde were at the same level, regardless of the animal's diet, which is in accordance with the TBARS level. These volatile aldehydes resulted from the oxidation of polyunsaturated fatty acids (PUFAs) and may be considered indicators of lipid oxidation for meat enriched with PUFAs. The results show that supplementation of the pigs' diet with linseed oil (L1 group), which is a source of PUFAs, is recommended for meat intended for long-term freezing storage. However, supplementation with antioxidants is unnecessary, because it has no effect on lipid oxidation of $L$. dorsi pork after long-term freezing storage.
\end{abstract}

Keywords: Animal's diet, frozen storage, lipid oxidation, volatile organic compounds

\#Corresponding author: marta_brodowska@sggw.pl

\section{Introduction}

Frozen storage is a widely used method of preserving meat and meat products for individual consumers and industries as it prolongs shelf life. Moreover, freezing reduces metabolic processes and nonenzymatic chemical changes, including oxidation. However, these mechanisms cannot be completely prevented by freezing (Huang et al., 2013). Gradual losses in the quality traits of meat occur as a result of an oxidative imbalance during long-term storage, even in a frozen state (Leygonie et al., 2012). Recently, swine feeding has been formulated with the addition of PUFAs, especially the $n-3$ series, to provide its higher concentration in meat. It is nutritionally desirable because of human health benefits (Kouba \& Mourot, 2011; Dugan et al., 2015), but implies increased susceptibility to oxidative processes (Sheard et al., 2000; Wojtasik-Kalinowska et al., 2016). The standard content in antioxidants is low in commercial pork (Reig \& Toldra, 1998). For this reason, it is necessary to supplement animal diets with antioxidant agents, such as polyphenol extracts, antioxidant vitamins and minerals, to reduce lipid oxidation in the resulting meat, especially in meat with a high content of PUFAs (O'Grady et al., 2001; Swigert et al., 2004; Descalzo et al., 2005; Lahucky et al., 2010). Previous studies suggested administering appropriate doses of vitamin $E$ and selenium to provide meat colour stability, limit changes in flavour, and prolong the shelf life of poultry, beef, 
lamb, and pork by inhibiting oxidation processes (Jensen et al., 1998; O'Grady et al., 2001; Descalzo et al., 2005; Perez et al., 2010; Vasta et al., 2013; Wojtasik-Kalinowska et al., 2016).

Flavour is a distinctive factor in determining meat quality. Flavour primarily defines its freshness and affects consumer palatability scores, and even consumers' decisions to purchase meat products (Park et al., 2008). The flavour of meat is strongly affected by animal diets and fodder additives (Vasta et al., 2006; Tikk et al., 2007; Vasta et al., 2013; Khan et al., 2015; Wojtasik-Kalinowska et al., 2016). The deterioration in the flavour of meat during storage occurs primarily because of oxidation of unsaturated fatty acids resulting from the deficiency of antioxidants. This leads to the appearance of lipid-derived volatile oxidation products with an unpleasant odour (Mottram, 1998; Leygonie et al., 2012; Vasta et al., 2013). Rancidity and the pungent odour of meat products are the results of the presence of saturated and unsaturated aldehydes or ketones. Although the levels of these compounds are generally low, sensory detection thresholds are significant compared with other volatile compounds. Supplementation with $n-3$ PUFAs may lead to the occurrence of other oxidation products and to changes in the total volatile compound profiles of meat. Consequently, the volatile compounds profile of meat products may be an indicator of meat oxidative processes (Descalzo et al., 2005).

There are not many studies on the oxidative stability and volatile profile of long-term frozen meat supplemented with PUFAs (Brodowska et al., 2016). Owing to a possible interaction between vitamin E and selenium and between these antioxidants and the freezing environment or tissue-specific muscle (O'Grady et al., 2001; Brodowska et al., 2016), an investigation of their activity is still required for successful application in meat products. The aim of this study was to analyse lipid oxidation and the volatile profile in lean pork meat ( $L$. dorsi muscle) derived from animals supplemented with linseed oil (as a source of $n-3$ PUFAs) and antioxidants (100 mg of vitamin E/ kg and $1 \mathrm{mg}$ of organic selenium $/ \mathrm{kg}$ of fodder) after long-term freezing storage.

\section{Materials and Methods}

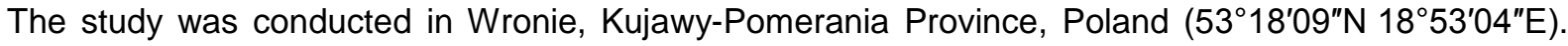
Thirty-six pigs (Polish Landrace $x$ Duroc), consisting of gilts and boars, were involved in the experiment in the project BIOFOOD - innovative, functional products of animal origin. Pigs ( 60 kg) were randomly allocated to three groups and fed with a standard diet (C) or diets containing bioactive components (L1, L2). Until $60 \pm 5 \mathrm{~kg}$ live weight was reached (the start of the experiment), all animals were fed with the standard fodder, which was $360 \mathrm{~g}$ barley grits $/ \mathrm{kg}, 360 \mathrm{~g}$ wheat middling $/ \mathrm{kg}, 100 \mathrm{~g}$ corn grits $/ \mathrm{kg}, 80 \mathrm{~g}$ extracted soybean meal $/ \mathrm{kg}, 40 \mathrm{~g}$ extracted rapeseed meal $/ \mathrm{kg}$ and $25 \mathrm{~g}$ vitamin-mineral premix $/ \mathrm{kg}$ as described previously (Guzek et al., 2012; Brodowska et al., 2016; Wojtasik-Kalinowska et al., 2016). After the experiment began, group $\mathrm{C}$ received a standard diet without additives; group L1 received a standard diet with $3 \%$ linseed oil; and group L2 received a standard diet with $3 \%$ linseed oil, $100 \mathrm{mg} / \mathrm{kg}$ vitamin $\mathrm{E}$, in the stable form of tocopheryl acetate and $1 \mathrm{mg}$ organic selenium $/ \mathrm{kg}$ of fodder. Animals from each group were slaughtered in industrial conditions in accordance with the current EU regulations after they reached $100 \pm 5$ $\mathrm{kg}$. The experimental procedures were approved by the local ethical commission (No. 27/2009). Animal carcasses belonged to $\mathrm{S}$ and $\mathrm{R}$ classes in the EUROP grading system. Six carcasses were randomly selected from each group, and six loins were separated from the right half-carcasses. Fresh, boneless loins (L. dorsi muscles) with a normal pH level $(5.61 \pm 0.15$ at 48 hours post mortem estimated by the potentiometric method using a pH electrode; Testo 205, Mera, Germany) were transported to the laboratory in refrigerated conditions. Meat analyses were conducted at the Laboratory of Food Chemistry of Warsaw University of Life Sciences (WULS-SGGW), Warsaw, Poland. Next, 18 loins were characterized by measurements of basic composition using a near-infrared spectrometer (NIRFlex Solids N-500, Büchi, Labortechnik Ltd., Germany), as presented in Table 1. Fresh sliced loins (approximately $150 \mathrm{~g}$ ) were vacuum packed and stored at a temperature of $-20^{\circ} \mathrm{C} \pm 1{ }^{\circ} \mathrm{C}$ for nine months for analysis.

Table 1 Basic composition of Longissimus dorsi samples of pork (mean \pm SD)

\begin{tabular}{lcccc}
\hline Feeding Groups & Moisture (\%) & Fat (\%) & Protein (\%) & Ash (\%) \\
\hline C & $72.70 \pm 1.60$ & $3.68 \pm 1.75$ & $23.26 \pm 0.49$ & $1.32 \pm 0.09$ \\
L1 & $73.19 \pm 1.05$ & $3.17 \pm 1.01$ & $23.42 \pm 0.24$ & $1.35 \pm 0.13$ \\
L2 & $71.84 \pm 1.03$ & $4.89 \pm 0.79$ & $22.98 \pm 0.31$ & $1.37 \pm 0.12$
\end{tabular}

C: control group, a standard diet; L1: a standard diet with the addition 3\% of linseed oil; L2: a standard diet with the addition $3 \%$ of linseed oil, $1 \mathrm{mg} / \mathrm{kg}$ organic selenium and $100 \mathrm{mg} / \mathrm{kg}$ vitamin $\mathrm{E}$ 
The oxidative stability analysis of the meat samples was carried out according to Brodowska et al. (2016). A sample (2.5 g) was homogenized with $25 \mathrm{ml}$ chilled trichloroacetic acid solution (200 g/l) and 1.25 $\mathrm{ml}$ synthetic antioxidant for about 30 seconds at 1200 rpm (WT 500 homogenizer, Wiggenhauser, Germany). After being centrifuged for $10 \mathrm{~min}$. at $8000 \mathrm{rpm}$ (centrifuge MPW-260, MPW Med. Instruments, Warsaw, Poland) $5 \mathrm{ml}$ 2-thiobarbituric acid $(0.02 \mathrm{mM} / \mathrm{l})$ was added to $5 \mathrm{ml}$ supernatant. The reactive mixtures were heated in a water bath $\left(90^{\circ} \mathrm{C}\right)$ for $40 \mathrm{~min}$ for a colour reaction. The absorbance was measured at $532 \mathrm{~nm}$, using the UV-VIS spectrophotometer (Shimazu UV-1800, Tokyo, Japan). The recovery of samples was conducted by analysis of the sample with an addition of standard (1,1,3,3-tetramethoxypropane) water solution $0.2 \mu \mathrm{M} / \mathrm{l}$. A calibration curve was evaluated with 1,1,3,3-tetramethoxypropane. Results were expressed as $\mathrm{mg}$ malondialdehyde/kg meat. The measurements were performed in triplicate for six loins of each diet group $(n=54 ; 3 \times 6 \times 3)$.

The volatile compounds of meat were measured with an Alpha MOS electronic nose system (Alpha MOS, Toulouse, France), according to Wojtasik-Kalinowska et al. (2016). The measurement was conducted using the ultra-fast gas chromatography system with two column (MXT-5 and MXT-1701; Restek, Munich, Germany) terminated flame ionization detectors, and the headspace technique. Alpha MOS software (Alpha Soft v. 8.0) was used for the acquisition and processing of the data. Vacuum-packaged samples were thawed at $4 \pm 1{ }^{\circ} \mathrm{C}$ for 16 hours. Samples of $2.5 \mathrm{~g}$ pork meat were placed in $20 \mathrm{ml}$ headspace vials and capped with a Teflon-faced silicon rubber cap. The vials were placed in the robotic autosampler of the headspace system. Each vial was incubated at $50{ }^{\circ} \mathrm{C}$ for $10 \mathrm{~min}$ under agitation (500 rpm). The injected volume was $3000 \mu \mathrm{l}$ with a speed of gas of $125 \mathrm{ml} / \mathrm{s}$ and the injector temperature was $200{ }^{\circ} \mathrm{C}$. Volatile compounds were identified based on Kovat's relative retention indices (alkanes from C6 to $\mathrm{C} 16$ ) with AroChemBase database (Alpha MOS). An analysis of volatile compounds was conducted in four replications for six loins of each group ( $n=72 ; 3 \times 6 \times 4)$.

The results are reported as the mean \pm SD or median with minimum and maximum values for TBARS and as the mean \pm SD for the relative peaks area of volatile aldehydes. The normality of data distribution was tested with the Shapiro-Wilk test. The differences among feeding groups were examined using MannWhitney's U Test in nonparametric distribution. Data with normal distribution were tested with one-way ANOVA (Tukey's post-hoc test). A level of significance $\alpha=0.05$ was taken. All analyses were performed using Statistica Software version 12.0, 2013 (StatSoft, Tulsa, Oklahoma, USA).

\section{Results and Discussion}

The data showed that long-term frozen storage did not stop the oxidative processes in any of the diets. The results revealed the appearance of PUFA decomposition products expressed as malondialdehyde in all experimental groups. The level of TBARS for each group of samples was approximately 2 mg malondialdehyde/kg of meat (Table 2). However, the impact of the addition of PUFAs or PUFAs and vitamin $\mathrm{E}$ and selenium on the animal fodder on lipid oxidation was insignificant at the defined fat level, exceeding 3\% (Table 1).

Table 2 Thiobarbituric acid reactive substance expressed as malondialdehyde values in Longissimus dorsi muscle of pork (mean \pm SD)

\begin{tabular}{lcc}
\hline & \multicolumn{2}{c}{ Malondialdehyde values (mg/kg) } \\
\cline { 2 - 3 } Feeding Groups & Mean \pm SD & Median (Min-Max) \\
\hline & $2.01^{\mathrm{a}} \pm 0.83$ & $2.64(0.97-4.81)$ \\
L1 & $2.30^{\mathrm{a}} \pm 1.10$ & $2.93(1.46-4.58)$ \\
L2 & $1.88^{\mathrm{a}} \pm 0.52$ & $1.81(1.37-3.29)$
\end{tabular}

\footnotetext{
a The same letters in the column indicate a lack of significant differences $(P>0.05)$

C: control group, a standard diet

L1: a standard diet with the addition $3 \%$ of linseed oil

L2: a standard diet with the addition $3 \%$ of linseed oil, $1 \mathrm{mg} / \mathrm{kg}$ organic selenium, and $100 \mathrm{mg} / \mathrm{kg}$ vitamin E
}

The presence of PUFAs in the phospholipid membrane is considered liable for the initiation of oxidation processes (Reis et al., 2012). In previous studies, Högberg et al. (2002) and Kouba \& Mourot (2011) found that diets rich in polyunsaturated fat increased susceptibility to lipid oxidation in pigs' $L$. dorsi 
muscles and resulted in a decrease in oxidative stability, in particular after frozen storage (Vieira et al., 2009). A different scientific approach was presented by Perez et al. (2010), who showed that the time of storage had the biggest impact on the efficacy of antioxidant supplementation in preventing lipid oxidation in frozen meat. In their study, dietary vitamin E in doses of $250 \mathrm{IU} / \mathrm{kg}(167.5 \mathrm{mg} / \mathrm{kg})$ and selenium in the form of $0.3 \mathrm{mg}$ selenomethionine $/ \mathrm{kg}$ and $0.1 \mathrm{mg}$ sodium selenite $/ \mathrm{kg}$ of fodder inhibited lipid oxidation product formation in poultry after six months of frozen storage, even with high levels of PUFAs. In contrast, no dietary antioxidant protection was observed in raw poultry after 12 months of storage at $-30{ }^{\circ} \mathrm{C}$ (Perez et al., 2010). In the present study, after nine months of storage at $-20^{\circ} \mathrm{C}$, the effect of the diet on lipid oxidation was negligible.

In other authors' research, molecules such as a-tocopherol (the dominant form of vitamin E) had no effect on the decrease in oxidation during long-term storage in freezing conditions (Channon \& Trout, 2002; Brodowska et al., 2016). In the present study, the lack of effect of antioxidants on meat stability could be explained by the low levels of $\alpha$-tocopherols in meat after long-term storage. The storage result in depletion of endogenous antioxidants (Perez et al., 2010) or the decrease in their molecular mobility during frozen storage (Niki \& Noguchi, 2004). O'Grady et al. (2001) suggested that dietary selenium combined with $\alpha$ tocopherol had high potential for increasing oxidative defence. However, in the time of freezing storage, the low activity of $\alpha$-tocopherol renders selenium action ineffective in preventing lipid oxidation. The relatively high total fat content, especially of PUFAs, and the low content of fat-soluble antioxidants such as vitamin E are responsible for meat stability problems. Therefore, long-term freezing did not provide the antioxidant effect of fodder supplementation on lipid oxidation in the obtained meat. Similar results were obtained for another pork muscle, Semimembranosus, stored for nine months at $-20^{\circ} \mathrm{C}$ (Brodowska et al., 2016). On the other hand, the lack of effect of diet on pork quality may be considered desirable. It shows that supplementation with linseed oil as a source of $n-3$ PUFAs did not lead to the oxidative deterioration of lipids compared with control after nine months of freezing storage. This is associated with the slow oxidation process, the depletion of endogenous antioxidants, and with fodder enrichment with antioxidants, as mentioned above. Similarly, Sheard et al. (2000) presented no adverse effects of PUFAs supplementation on the appearance of lipid oxidation products in loin chops (from $L$. dorsi).

The volatile compounds detected in $L$. dorsi pork after nine months of storage are typical of pork meat and lipid oxidation (Table 3). This is consistent with other studies (Wojtasik-Kalinowska et al., 2016; GórskaHorczyczak et al., 2017). Previously, butanal, 1-propanol and methyl hexanoate were detected in raw loins derived from pigs fed diets with the addition of linseed oil and in loins derived from pigs fed diets with the addition of linseed oil, vitamin $\mathrm{E}$, and selenium. Benzeneacetaldehyde in raw loins was detected in the group supplemented only with linseed oil (Wojtasik-Kalinowska et al., 2016). In comparison with the current research, this suggested that $100 \mathrm{mg}$ vitamin E/kg and $1 \mathrm{mg}$ selenium/ $\mathrm{kg}$ of pig fodder reduces the appearance of aldehyde compounds in fresh (non-stored) meat, but not in frozen meat. According to GórskaHorczyczak et al. (2017), the profile of VOC from the frozen stored meat consisted mostly of aldehydes. Linear aldehydes are considered important products of lipid peroxide decomposition because they possess low threshold values and are related to the production of undesirable odours (Lozano et al., 2007). Volatile compounds that are oxidation-derived aldehydes represented approximately $10 \%, 12 \%, 15 \%$ of total detected volatiles for L2, L1 and C groups, respectively. However, differences in the sum of aldehydes of each diet group were not significant. No effects of dietary treatment on the volatile aldehyde profile were reported in dry-cured ham derived from pigs fed various monounsaturated fatty acids /PUFAs ratios and vitamin E (Isabel et al., 2003). However, other authors found that a change only in the specific unsaturated fatty acid composition had an influence on the pork loin flavour (Tikk et al., 2007; Aaslyng et al., 2008). Dietary antioxidants seem to have an effect on the volatile compound profile of meat but no effect on lipid oxidation.

In the study, three aldehydes, namely propanal, benzeneacetaldehyde and butanal, were identified in all samples from analysed feeding groups (C, L1 and L2) of the $L$. dorsi muscle. With this in mind, relative peak areas were analysed to determine the level of aldehydes in groups. Supplemented groups differed in 2-methylpropanal and butanal levels. In propanal and benzeneacetaldehyde, the differences in relative peak area were insignificant (Figure 1). A higher content of butanal in the C group compared with L1 and L2 groups may indicate a progress of oxidation processes. Furthermore, 2-methylpropanal was not identified in the L1 group. In the L2 group, its content was lower compared with the control. 2-methylpropanal is described as having a burnt and pungent odour resulting from the catabolism of branched chain amino acids (Yvon et al., 2001). 
Table 3 Volatile compounds identified in Longissimus dorsi muscle by electronic nose

\begin{tabular}{lccc}
\hline $\begin{array}{l}\text { Chemical group of } \\
\text { compounds }\end{array}$ & Volatile compound & Odour descriptions* & $\begin{array}{c}\text { Feeding } \\
\text { groups }\end{array}$ \\
\hline $\begin{array}{l}\text { amines } \\
\text { aldehydes }\end{array}$ & $\begin{array}{c}\text { trimethyloamina } \\
\text { propanal }\end{array}$ & ammoniacal, fishy, pungent & C, L1, L2 \\
& 2-methyl-propanal & ethereal, plastic, pungent & C, L1, L2 \\
& benzeneacetaldehyde & burnt, pungent & C, L2 \\
& butanal & floral, grassy, green, hawthorn & C, L1, L2, \\
alkanes & nonane & chocolate, green, malty, pungent & C, L1, L2 \\
alcohols & methanol & alkane, fusel & L1 \\
& 1-propanol & alcoholic, pungent & C, L1, L2 \\
& 3-methyl-1-butanol & alcoholic, balsamic, bitter, burnt, cheese, fermented, & C, L1, L2 \\
esters & fruity, harsh, malty & L1 \\
& methyl butanoate & ester, ethereal, fruity, green, sweet, & C, L1, L2 \\
& methyl isobutyrate & nutty & L1, L2
\end{tabular}

C: control group, standard diet

L1: group I, a standard diet with the addition of $3 \%$ linseed oil

L2: group II, a standard diet with the addition of $3 \%$ linseed oil, $1 \mathrm{mg} \cdot \mathrm{kg}^{-1}$ organic selenium, and $100 \mathrm{mg} \cdot \mathrm{kg}^{-1} \mathrm{vitamin} \mathrm{E}$

* Characterized according to AroChemBase database for compounds separated by gas chromatography

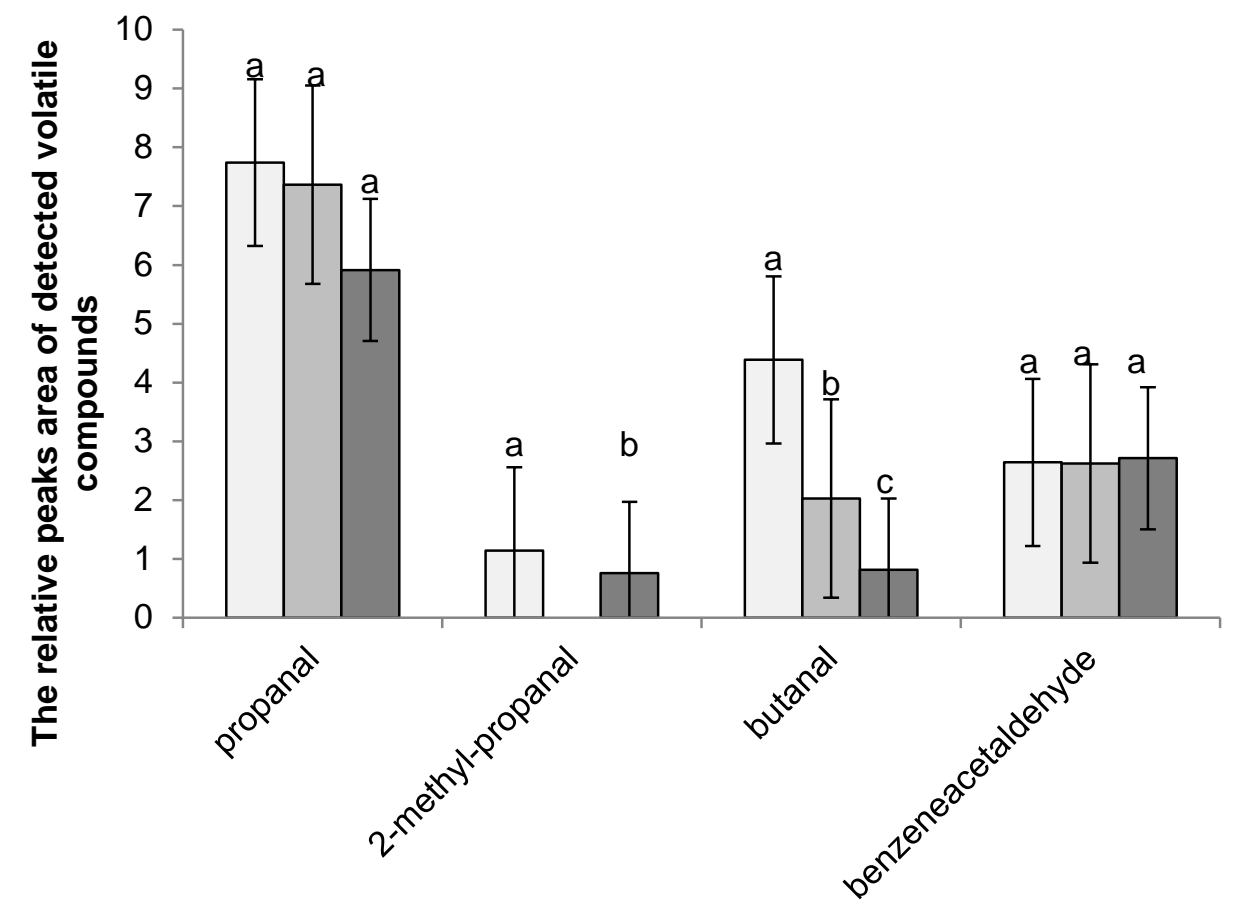

$\square \mathrm{C}$

口L1

*a, b, c: different letters in the column indicate significant differences $(P \leq 0.05)$

C: control group, standard diet

L1: a standard diet with the addition of $3 \%$ linseed oil

L2: a standard diet with the addition of $3 \%$ linseed oil, $1 \mathrm{mg} / \mathrm{kg}$ organic selenium, and $100 \mathrm{mg} / \mathrm{kg}$ vitamin E

Figure 1 Lipid-derived organic volatile compounds identified in Longissimus dorsi pork (relative area of peaks, mean $\pm \mathrm{SD}$ ) 
However, owing to the high reactivity of aldehydes with other compounds, aldehydes undergo further transformation. The reduction of some aldehydes leads to the formation of alcohols, for example 3-methyl-1butanol, and esters, for example methyl butanoate (Leroy et al., 2009), which was established in the study (Table 3). Park et al. (2008) found that 3-methyl-1-butanol level increased during pork loin storage. It suggests that alcohols and esters may be indirect indicators of the oxidation process. Methanol and methyl butanoate were identified only in L1 group. This suggests that the supplementation of linseed oil introduced new substances or precursors of volatile compounds.

Typical retail pork suffers from an imbalance of the $n-6 / n-3$ fatty acid ratio. Pork is too abundant in the contents of $n-6$ PUFAs compared with $n$-3 PUFAs (Dugan et al., 2015). Supplementation of animal diets with $n$-3 PUFAs not only increased $n-3$ PUFAs in intramuscular fat, even in lean muscles such as $L$. dorsi, but also modified the flavour profile (Khan et al., 2015; Wojtasik-Kalinowska et al., 2016). Volatile hexanal is frequently considered an indicator of lipid oxidation for meat-based products, and is generated in cooked meat in particular as a product of $n-6$ PUFAs, linoleic acid oxidation (Pignoli et al., 2009). However, besides hexanal, volatile compounds such as propanal and octanal are chosen as markers of deterioration in products with high fat content (including oils, meat products, and bakery products), depending on the fatty acid profile. In the current study, benzeneacetaldehyde occurred in each group. Similarly, propanal occurred with no relation to the fodder supplementation. Benzeneacetaldehyde is created from the oxidative decomposition of $n$-6 PUFAs (Huang et al., 2013). In turn, propanal derived from $n$-3 PUFAs (alpha-linolenic, eicosapentaenoic and docosahexaenoic acids) breakdown (Romeu-Nadal et al., 2004; Pignoli et al., 2009). Therefore, in meat supplemented with linseed oil ( $n-3$ PUFA enriched product), propanal seems to be a more appropriate indicator of oxidation. The content of a single aldehyde can vary between the analysed feeding groups (2-methylpropanal and butanal). The total content of aldehydes and propanal and benzeneacetaldehyde in the groups was in accordance with the content of TBARS expressed by malondialdehyde. The compatibility of the results indicates that the determination of propanal and benzeneacetaldehyde, which are proposed indicators of the lipidic oxidation state, may be recommended for rapid evaluation of lipid oxidation in pork meat after freezing storage.

\section{Conclusions}

The effect of linseed oil or linseed oil and antioxidants (100 mg vitamin E/kg and $1 \mathrm{mg}$ organic selenium $/ \mathrm{kg}$ of fodder) on oxidative stability was insignificant in $L$. dorsi pork (with fat content $>3 \%$ ) after nine months of the storage. The profile of volatile compounds of meat after freezing storage vary between diet groups. However, propanal, and benzeneacetaldehyde and furthermore the total content of aldehydes did not vary between diet groups.

The data indicated that supplementation of pig diets with linseed oil, which is a source of PUFAs, is recommended for pork that is intended for long-term freezing storage. However, supplementation of pig diets with antioxidants is unnecessary. Moreover, the use of volatile aldehydes such as propanal, and benzeneacetaldehyde may be recommended for the evaluation of the lipid oxidation of meat obtained from pigs after long-term storage.

\section{Acknowledgements}

Research financed by Polish Ministry of Science and Higher Education from funds of Faculty of Human Nutrition and Consumer Sciences, Warsaw University of Life Sciences (WULS-SGGW), for scientific research.

This work was supported by the Project Bio Food Innovative, Functional Products Of Animal Origin no.

POIG.01.01.02-014-090/09 co-financed by the European Union from the European Regional Development Fund in the Innovative Economy Operational Programme 2007-2013.

\section{Authors' Contributions}

MB, DG \& AW designed the study. MB conducted the laboratory analysis, data analysis and manuscript writing. DG \& JG were involved in the interpretation of the data and constructive revision of the manuscript. EP participated in interpretation of the data.

\section{Conflict of interest declaration}

The authors declare that they have no competing interests.

\section{References}

Aaslyng, M.D. \& Schäfer, A., 2008. The effect of free fatty acids on the odour of pork investigated by sensory profiling and GC-O-MS. Eur. Food Res. Technol. 226, 937-948. 
Brodowska, M., Guzek, D., Kołota, A., Głąbska, D., Górska-Horczyczak, E., Wojtasik-Kalinowska, I. \& Wierzbicka, A., 2016. Effect of diet on oxidation and profile of volatile compounds of pork after freezing storage. J. Food Nutr. Res. 55, 40-47.

Channon, H.A. \& Trout, G.R., 2002. Effect of tocopherol concentration on rancidity development during frozen storage of a cured and an uncured processed pork product. Meat Sci. 62, 1, 9-17.

Descalzo, A.M., Insani, E.M., Biolatto, A., Sancho, A.M., García, P.T., Pensel, N.A. \& Josifovich, J.A., 2005. Influence of pasture or grain-based diets supplemented with vitamin $E$ on antioxidant/oxidative balance of Argentine beef. Meat Sci. 70, 1, 35-44.

Dugan, M.E.R., Vahmani, P., Turner, T.D., Mapiye, C., Juárez, M., Prieto, N., Beaulieu, A.D., Zijlstra, R.T., Patience J.F. \& Aalhus, J.L., 2015. Pork as a source of omega-3 (n-3) fatty acids. J. Clin. Med. 4, 12, 1999-2011.

Górska-Horczyczak, E., Wojtasik-Kalinowska, I., Guzek, D., Sun, D.-W. \& Wierzbicka, A., 2017. Differentiation of chillstored and frozen pork necks using electronic nose with ultra-fast gas chromatography. J. Food Process Eng. 40, 5 , e12540.

Guzek, D., Głąbska, D., Sakowska, A. \& Wierzbicka, A., 2012. Color of smoked loin from animals fed with bioactive compounds added to forage. Pesq. Agropec. Bras. 47, 1504-1510.

Högberg, A., Pickova, J., Babol, J., Andersson, K. \& Dutta, P.C., 2002. Muscle lipids, vitamins E and A, and lipid oxidation as affected by diet and RN genotype in female and castrated male Hampshire crossbreed pigs. Meat Sci. 60, 4, 411-420.

Huang, L., Xiong, Y.L., Kong, B., Huang, X. \& Li, J., 2013. Influence of storage temperature and duration on lipid and protein oxidation and flavour changes in frozen pork dumpling filler. Meat Sci. 95, 295-301.

Isabel, B., Lopez-Bote, C.J., de la Hoz, L., Timón, M., García, C. \& Ruiz, J., 2003. Effects of feeding elevated concentrations of monounsaturated fatty acids and vitamin $E$ to swine on characteristics of dry cured hams. Meat Sci. 64, 475-482.

Jensen, C., Lauridsen, C. \& Bertelsen, G., 1998. Dietary vitamin E: Quality and storage stability of pork and poultry. Trends Food Sci. Technol. 9, 62-72.

Khan, M.I., Jo, C. \& Tariq, M.R., 2015. Meat flavor precursors and factors influencing flavor precursors - A systematic review. Meat Sci. 110, 278-284

Kouba, M. \& Mourot, J., 2011. A review of nutritional effects on fat composition of animal products with special emphasis on n-3 polyunsaturated fatty acids. Biochimie. 93, 1, 13-17.

Lahucky, R., Nuernberg, K., Kovac, L., Bucko, O. \& Nuernberg, G., 2010. Assessment of the antioxidant potential of selected plant extracts - In vitro and in vivo experiments on pork. Meat Sci. 85, 4, 779-784.

Leroy, F., Vasilopoulos, C., Van Hemelryck, S., Falcony, G. \& De Vuyst, L., 2009. Volatile analysis of spoiled, artisantype, modified-atmosphere-packaged cooked ham stored under different temperatures. Food Microbiol. 26, 94-102.

Leygonie, C., Britz, T.J. \& Hoffman, C.L., 2012. Impact of freezing and thawing on the quality of meat: Review. Meat Sci. 91, 93-98.

Lozano, P.R., Miracle, E.R., Krause, A.J., Drake, M. \& Cadwallader, K.R., 2007. Effect of cold storage and packaging material on the major aroma components of sweet cream butter. J. Agri. Food Chem. 55, 7840-7846.

Mottram, D.S., 1998. Flavour formation in meat and meat products: A review. Food Chem. 62, 415-424.

Niki, E. \& Noguchi, N., 2004. Dynamics of antioxidant action of vitamin E. Accounts Chem. Research, 37, 45-51.

O'Grady, M.N., Monahan, F.J., Fallon, R.J. \& Allen, P., 2001. Effects of dietary supplementation with vitamin E and organic selenium on the oxidative stability of beef. J. Anim. Sci. 79, 2827-2834.

Park, S.Y., Kim, Y.J., Lee, H.C., Yoo, S.S., Shim, J.H. \& Chin, K.B., 2008. Effects of pork meat cut and packaging type on lipid oxidation and oxidative products during refrigerated storage $\left(8^{\circ} \mathrm{C}\right)$. J. Food Sci. 73, 3, 127-134.

Perez, T.I., Zuidhof, M.J., Renema, R.A., Curtis, J.M., Ren, Y. \& Betti, M., 2010. Effects of vitamin E and organic selenium on oxidative stability of omega-3 enriched dark chicken meat during cooking. J. Food Sci. 75, 2, 25-34.

Pignoli, G., Bou, R., Rodrigez-Estrada, M.T. \& Decker, E.A., 2009. Suitability of saturated aldehydes as lipid oxidation markers in washed turkey meat. Meat Sci. 83, 412-416.

Reig, M. \& Toldrá, F., 1998. Pork meat as a source of vitamins. Recent Res. Devel. Nutr. 2, 29-34.

Reis, A. \& Spickett, C.M., 2012. Chemistry of phospholipid oxidation. BBA Biomembranes. 1818, 10, $2374-2387$.

Romeu-Nadal, M., Castellote, A.I. \& López-Sabater, M.C., 2004. Headspace gas chromatographic method for determining volatile compounds in infant formulas. J. Chromatogr. A. 1046, 235-239.

Sheard, P.R., Enser, M., Wood, J.D., Nute, G.R., Gill, B.P. \& Richardson R.I., 2000. Shelf life and quality of pork and pork products with raised n-3 PUFA. Meat Sci. 55, 213-221.

Swigert, K.S., McKeith, F.K., Carr, T.C., Brewer, M.S. \& Culbertson, M., 2004. Effects of dietary vitamin D3, vitamin E, and magnesium supplementation on pork quality. Meat Sci. 67, 81-86.

Tikk, K., Tikk, M., Aaslyng, M.D., Karlsson, A.H., Lindahl, G. \& Andersen, H.J., 2007. Significance of fat supplemented diets on pork quality - Connections between specific fatty acids and sensory attributes of pork. Meat Sci. 77 , 275-286.

Vasta, V. \& Priolo, A., 2006. Ruminant fat volatiles as affected by diet. A review. Meat Sci. 73, 2, 218-228.

Vasta, V., Aouadi, D., Brogna, D.M.R., Scerra, M., Luciano, G., Priolo, A. \& Salem, H.B., 2013. Effect of the dietary supplementation of essential oils from rosemary and artemisia on muscle fatty acids and volatile compound profiles in Barbarine lambs. Meat Sci. 95, 2, 235-241.

Vieira, C., Diaz, M.T., Martínez, B. \& García-Cachán, M.D., 2009. Effect of frozen storage conditions (temperature and length of storage) on microbiological and sensory quality of rustic crossbred beef at different states of ageing. Meat Sci. 83, 3, 398-404. 
Wojtasik-Kalinowska, I., Guzek, D., Górska-Horczyczak, E., Głąbska, D., Brodowska, M., Sun, D-W. \& Wierzbicka, A., 2016. Volatile compounds and fatty acids profile in longissimus dorsi muscle from pigs fed with feed containing bioactive components. LWT-Food Sci. Technol. 67, 112-117.

Yvon, M. \& Rijnen, L., 2001. Cheese flavour formation by amino acid catabolism. Int. Dairy J. 11, 185-201. 